\title{
Quality of life and deglutition after total laryngectomy
}

\author{
Leandro de Araújo Pernambuco', Jabson Herber Profiro de Oliveira², Renata Milena Freitas Lima Régis³, Leilane Maria de \\ Lima $^{2}$, Ana Maria Bezerra de Araújo ${ }^{4}$, Patrícia Maria Mendes Balata ${ }^{5}$, Daniele Andrade da Cunha ${ }^{6}$, Hilton Justino da Silva ${ }^{7}$.
}

1) Master in Health Sciences - Federal University of Pernambuco. Professor - Speech, Language and Hearing Sciences Department - Federal University of Rio Grande do Norte.

2) Graduated in Speech, Language and Hearing Science - Federal University of Pernambuco. Speech and Language Pathologist.

3) Specialist in Orofacial Miology. Speech and Language Pathologist.

4) Specialist in Dysphagia - Federal Council of Speech, Language and Hearing Sciences. Speech and Language Pathologist - Cancer Hospital of Pernambuco.

5) Master of Science in Adolescent Health - Pernambuco University. Speech and Language Pathologist. Institute of Human Resources of Pernambuco State.

6) Ph.D in Nutrition - Federal University of Pernambuco. Professor - Estacio Recife.

7) Ph.D in Nutrition - Federal University of Pernambuco. Professor - Speech, Language and Hearing Science Department - Federal University of Pernambuco.

Institution: Universidade Federal de Pernambuco.

Recife / PE - Brazil

Mailing address: Leandro de Araújo Pernambuco - Departamento de Fonoaudiologia - Rua General Gustavo Cordeiro de Farias, s/n - Petrópolis - Natal / RN - Brazil Zip code: 59010-180 - Telephone: (+55 84) 3342-9738 - E-mail: leandroape@ globo.com

Conselho Nacional de Tecnologia e Desenvolvimento Científico (CNPq) - Edital MCT/CNPq/CT-Saúde/MS/SCTIE/DECIT nº 67/2009 - REBRATS

Article received in April 8, 2012. Article approved in August 10, 2012.

\section{SUMMARY}

Introduction: Total laryngectomy creates deglutition disorders and causes a decrease in quality of life

Aim: To describe the impact of swallowing and quality of life of patients after total laryngectomy.

Method: A case series study. Patients completed a Swallowing and Quality of Life questionnaire composed of 44 questions assessing 11 domains related to quality of life (burden, eating duration, eating desire, frequency of symptoms, food selection, communication, fear, mental health, social functioning, sleep, and fatigue). The analysis was performed using descriptive statistics, including measures of central tendency and variability.

Results: The sample comprised 15 patients who underwent total laryngectomy and adjuvant radiotherapy. Of these, 66.7\% classified their health as good and $73 \%$ reported no restrictions on food consistency. The domains "communication" and "fear" represented severe impact and "eating duration" represented moderate impact on quality of life. The items with lower scores were: longer time to eat than others (domain "eating duration"), cough and cough to remove the liquid or food of the mouth when they are stopped (domain "symptom frequency"), difficulties in understanding (domain "communication") and fear of choking and having pneumonia (domain "fear").

Conclusion: After total laryngectomy, patients report that swallowing issues have moderate to severe impact in "communication," "fear," and "eating duration" domains.

Keywords: quality of life; laryngeal neoplasms; laryngectomy; deglutition; deglutition disorders.

\section{INTRODUCTION}

Recent proposals for treatment of advanced cancer of the larynx emphasize more conservative approaches and make the definition of treatment an even more complex (1). However, total laryngectomy is still frequently adopted in such cases and questionnaires on health conditions and quality of life have been recommended as key promoters of success of treatment planning (2).

After total laryngectomy, oropharyngeal dysphagia can compromise the quality of life by requiring the modification of eating habits, affect socialization, and lead to a degree of isolation in activities with family members (3). Oropharyngeal dysphagia is a common symptom in patients with tumors in the head and neck regions (4) and its etiology may be related to how surgical technique would be used, adjuvant treatments such as radiotherapy and chemotherapy, and comorbidities such as advanced age and depression $(5,6)$.

The impact of difficulty in deglutition in quality of life of total laryngectomized has been assessed using generic instruments $(3,7-9)$ or instruments specific to this function $(2,10,11)$. The results of these studies indicate that the overall quality of life after total laryngectomy is approaching the standard of the general population, as opposed to specific domains such as deglutition, which often appear associated with negative aspects (8). Nevertheless, the results are still preliminary, especially owing to the multiple number of existing instruments and because dysphagia is still underdiagnosed in this group of patients (5). The aim of this study was to describe the effect of deglutition in quality of life of patients undergoing total laryngectomy. 


\section{Method}

The participants included patients undergoing treatment for esophageal speech acquisition at the Department of Speech Therapy in a cancer referral center located in Pernambuco, northeastern part of Brazil. We included patients who underwent total laryngectomy with neck dissection and postoperative radiotherapy, with completion of treatment for at least 3 months. We excluded patients with neurological disorders and head and neck disease and those subjected to other procedures in the head and neck.

The quality of life related to deglutition was assessed using the Swallowing Quality of Life Questionnaire (SWALQOL), which has been validated for Brazilian Portuguese subjects (12). The questionnaire comprises 44 questions that assess 11 domains related to quality of life (burden, feed duration, desire, symptoms frequency, food selection, communication, fear, mental health, social function, sleep, and fatigue). Each question has 5 possible answers. The answers were converted into scores ranging from 0 to 100, divided into quintiles ( 0 as a minimum score and 100 as a maximum positive score). In each domain, the score values regarding responses were summed and the result was divided by the number of questions in the domain, reaching the end score. The scores $0-49$ were interpreted as a severe impact, 50-70 as a moderate impact, and 71100 slight impact or no impact (10). The analysis was performed using descriptive statistics. Due to the final conversion of the SWAL-QOL results into categories (ordinal categorical variable), we used the median as a measure of central tendency and the minimum and maximum as a measure of dispersion. Among the issues addressed by the additional instrument, we herein highlight the general health status and self-reported and food consistencies accepted by the volunteer. These variables were analyzed by means of absolute and relative frequencies.

The research was submitted to the Ethics and Human Research committee and approved under the number 67/2010. The volunteers who agreed to participate in the study signed a free consent term, in accordance to Resolution 196/96 of the National Research Ethics Counsel.

\section{RESULTS}

A sample of 15 volunteers had a mean age of 63 \pm 9.3 years and the following profile: male (86.7\%), married (53.3\%) and uncompleted elementary education (60\%). The self-reported condition general health was rated as good by a significant percentage of volunteers (Graphic 1).

Graphic 2 shows that most participants described the consistency of the food consumed in the last week as difficult to chew. No volunteer used any alternative feeding method at the time of data collection.

Table 1 shows the distribution of SWAL-QOL domains, according to the median. It was observed that the "communication" and "fear" domains were those with the lowest scores, indicating severe impact on quality of life related to deglutition. The "feeding duration" domain caused moderate impact and the others domains caused discrete impact or no impact.

The descriptive analysis (Chart 1) of data revealed that items with higher absolute and relative frequency of responses with scores between 0 and 50 were longer time required to eat (53.3\%; "feeding duration" domain), cough to remove the liquid or food out of the mouth when they are standing (40\% and 46.7\%, respectively, "frequency of symptoms" domain), difficult to understand (46.7\%; "communication" domain), and fear choking and having pneumonia (40\% and 53.3\%, respectively; "fear" domain).

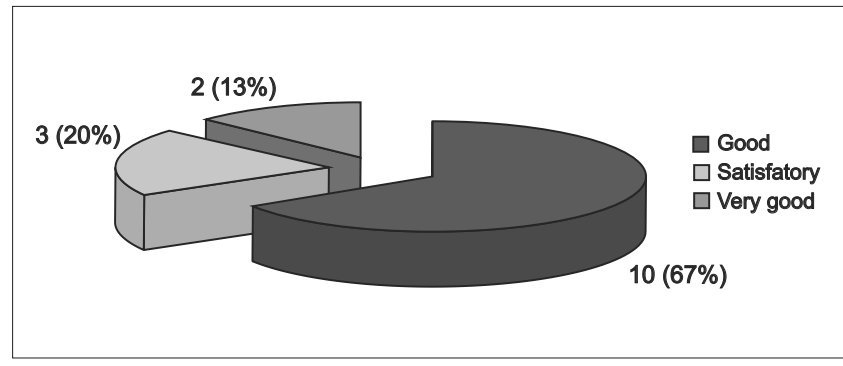

Chart 1. Overall health condition self-reported by patients with total laryngectomy. Quality of life relation to deglutition, Recife, 2011.

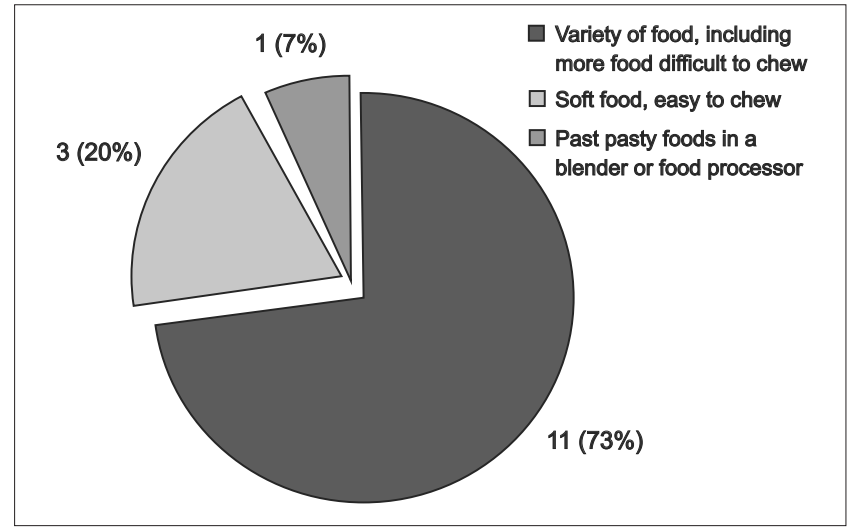

Chart 2. Graph of consistency or texture most common last week. Quality of life related to total laryngectomized deglutition, Recife, 2011. 
Table I. Distribution of variables related to SWAL-QOL domains, according to measures of central tendency and variability. Deglutition quality of liferelated to total laryngectomy. Recife, 20II.

\begin{tabular}{lcc}
\hline Variable & Median & Minimum-Maximum \\
\hline Deglutitionasaburden & 100 & $0-100$ \\
Feedingduration & 50 & $0-100$ \\
Desire to eat & 75 & $16.6-100$ \\
Symptomsfrequency & 80.3 & $37.5-96.4$ \\
Foodselection & 100 & $50-100$ \\
Comunication & 37.5 & $25-37.5$ \\
Fear & 43.7 & $25-43.7$ \\
Mental health & 95 & $70-95$ \\
Socialfunction & 95 & $25-95$ \\
Sleep & 75 & $37.5-75$ \\
Fatigue & 83.3 & $50-83.3$ \\
\hline
\end{tabular}

\section{DisCUSSION}

In Brazil, a group of researchers published 2 studies in which the SWAL-QOL questionnaire was used in patients after a total laryngectomy in São Paulo; however, unlike the present study, they found only a moderate impact of deglutition on quality of life $(10,11)$.

In one of these studies, 12 patients with total laryngectomies answered the questionnaire. In this sample, the domains that resulted in the lowest median values were "communication" and "desire for food," whose impact was moderate on the quality of life. It is worth noting that the "feeding duration" domain was not considered in this study. In the other work with a sample of the same health service, the lowest median found in the "feeding duration" and "communication" domains, with moderate impact on quality of life (11).

It is noticed that the "communication" domain is always present and associated with negative aspects. The permanent loss of laryngeal voice and the difficulties of adapting to alternative communication can support this result (13). The fact that the volunteers of this research are not yet fully adapted to esophageal speech may have influenced the result. In the institution where the collection was made, the esophageal voice was the method of communication rehabilitation, since the other possibilities of more sophisticated vocal rehabilitation (tracheoesophageal and laryngeal electronic prostheses) require costs that are not compatible with the profile of low-income users of this service.

We emphasize that the individual variations in relation to culture, beliefs, religions, social, economic, professional, and family situations (10) can exert a strong influence on the perception that the individuals have of their quality of life. Thus, the differences and similarities between our study and others may possibly be explained by the variable aspects of social determinants, whose importance should be further explored in future research.

The SWAL-QOL is a protocol that considers a specific functional domain; however, there are protocols that assess the overall quality of life and include deglutition $(3,9)$.

An Australian study (3) investigated the effect of dysphagia on quality of life of 110 patients after total laryngectomy. This study used the World Health Organization Quality of Life-BRIEF (WHOQOL-BRIEF) and the University of Washington QOL (UW-QOL). There was no difference between the findings of the subjects with and without dysphagia; however, total laryngectomy with dysphagia hadmore functional impairment, reduced social participation, and higher levels of depression and anxiety. The authors conclude that although dysphagia does not directly determine the quality of life after total laryngectomy, it can have a negative impact on functional and psychological wellbeing of the patient.

In another study (8) in patients with total laryngectomy with more than 2 years of completion of treatment, the overall quality of life did not differ from that of the general population, but there was adherence to specific scales of the physical domain, which is influenced by age, sex, radiotherapy, and chemotherapy. In the sample of these authors, women reported more difficulties with deglutition, which corroborates the findings of another study (14). Because of our small sample size, we did not compare the results by gender.

When considering the patient's perspective regarding the impact of total laryngectomy on quality of life, a qualitative study (15) received reports of psychological and functional problems, including dysphagia. The authors highlight the high number of difficulties reported even after end of treatment and reinforce the need for maintenance of the monitoring team of multidisciplinary rehabilitation for longer periods after surgery.

About this, the literature says that the longer survival is not an accurate reflection of the success of treatment and does not necessarily indicate better quality of life (2). Therefore, there is a need to stimulate a long-term care to these individuals and to promote the application of more reliable instruments that can capture the impression of the subject with respect to their quality of life.

For longer-term evaluations, the Performance Status Scale for Head and Neck Cancer Patients (PSS-HN) was 
Table 2. Distribution of the sample of patients with total laryngectomy according to the responses of SWAL-QOL questionnaire (Portas, 2009) with respect to burden, feeding duration, eating desire, symptom frequency, food selection, communication, fear, mental health, social function, sleep, and fatigue domains from quality of life related to deglutition in total laryngectomy patients (Recife20II).

\begin{tabular}{|c|c|c|c|c|c|}
\hline RESPONSES & $\begin{array}{c}\text { SCORE } 0 \\
\mathrm{n}(\%)\end{array}$ & $\begin{array}{c}\text { SCORE } 25 \\
n(\%)\end{array}$ & $\begin{array}{c}\text { SCORE } 50 \\
n(\%)\end{array}$ & $\begin{array}{c}\text { SCORE } 75 \\
n(\%)\end{array}$ & $\begin{array}{c}\text { SCORE } 100 \\
n(\%)\end{array}$ \\
\hline \multicolumn{6}{|l|}{ Deglutition as a burden domain } \\
\hline Dealing with my deglutition problem is very difficult & $3(20 \%)$ & - & - & $4(26.7 \%)$ & $7(46.7 \%)$ \\
\hline My deglutition problem is the major disruption in my life & I (6.7\%) & - & $2(13.3 \%)$ & & $12(80 \%)$ \\
\hline \multicolumn{6}{|l|}{ Feeding duration domain } \\
\hline It takes me longer to eat than others & $8(53.3 \%)$ & - & $5(33.3 \%)$ & I (6.7\%) & I (6.7\%) \\
\hline I take much time to eat my meal & $5(33.3 \%)$ & - & I'(6.7\%) & - & $9(60 \%)$ \\
\hline \multicolumn{6}{|l|}{ Eating desire domain } \\
\hline Most days, I do not care if I eat or not & $3(20 \%)$ & $2(13.3 \%)$ & - & $3(20 \%)$ & $7(46.7 \%)$ \\
\hline I'm rarely hungry & I (6.7\%) & $2(13.3 \%)$ & $2(13.3 \%)$ & $3(20 \%)$ & $7(46.7 \%)$ \\
\hline I don't have more pleasure in eating & $2(13.3 \%)$ & $2(13.3 \%)$ & $2(13.3 \%)$ & I (6.7\%) & $8(53.3 \%)$ \\
\hline \multicolumn{6}{|l|}{ Symptoms frequency domain } \\
\hline Cough & I (6.7\%) & $3(20 \%)$ & $6(40 \%)$ & $2(13.3 \%)$ & $3(20 \%)$ \\
\hline Choking when eating & - & I (6.7\%) & I (6.7\%) & $4(26.7 \%)$ & $9(60 \%)$ \\
\hline Choking with liquids & - & I (6.7\%) & - & $2(13.3 \%)$ & $12(80 \%)$ \\
\hline Present thick saliva or secretion & $3(20 \%)$ & $3(20 \%)$ & $4(26.7 \%)$ & I (6.7\%) & $4(26.7 \%)$ \\
\hline Vomiting & - & - & - & I (6.7\%) & $14(93.3 \%)$ \\
\hline Nausea & - & - & - & I (6.7\%) & $14(93.3 \%)$ \\
\hline Difficulties in chewing & $3(20 \%)$ & $2(13.3 \%)$ & - & - & $10(66.7 \%)$ \\
\hline Excessive secretion of saliva & $4(26.7 \%)$ & $2(13.3 \%)$ & $2(13.3 \%)$ & I (6.7\%) & $6(40 \%)$ \\
\hline Hawking & $2(13.3 \%)$ & | (6.7\%) & I (6.7\%) & - & $10(66.7 \%)$ \\
\hline The food stops in the throat & I (6.7\%) & I (6.7\%) & $3(20 \%)$ & $2(13.3 \%)$ & $8(53.3 \%)$ \\
\hline The food stops in the mouth & - & I (6.7\%) & I (6.7\%) & - & $13(86.7 \%)$ \\
\hline Food or drink drip from the mouth & I (6.7\%) & I (6.7\%) & - & I (6.7\%) & $12(80 \%)$ \\
\hline Food or drink out through the nose & - & I (6.7\%) & $3(20 \%)$ & I (6.7\%) & $10(66.7 \%)$ \\
\hline $\begin{array}{l}\text { Cough to remove liquid or food out of the mouth when } \\
\text { they are stopped }\end{array}$ & $7(46.7 \%)$ & r & $\mid(6.7 \%)$ & $2(13.3 \%)$ & $5(33.3 \%)$ \\
\hline \multicolumn{6}{|l|}{ Food selection domain } \\
\hline Knowing what I can and not can eat is a problem for me & $3(20 \%)$ & - & I (6.7\%) & $3(20 \%)$ & $8(53.3 \%)$ \\
\hline $\begin{array}{l}\text { It's hard to find foods I can and like to eat } \\
\text { Communication domain }\end{array}$ & - & - & I (6.7\%) & I (6.7\%) & $13(86.7 \%)$ \\
\hline People have difficulty understanding me & I (6.7\%) & $7(46.7 \%)$ & $5(33.3 \%)$ & - & $2(13.3 \%)$ \\
\hline It has been difficult to communicate clearly & $4(26.7 \%)$ & $3(20 \%)$ & $4(26.7 \%)$ & $2(13.3 \%)$ & $2(13.3 \%)$ \\
\hline \multicolumn{6}{|l|}{ Fear domain } \\
\hline I'm afraid of choking when I eat & $6(40 \%)$ & I (6.7\%) & $2(13.3 \%)$ & I (6.7\%) & $5(33.3 \%)$ \\
\hline I worry about having pneumonia & $8(53.3 \%)$ & I (6.7\%) & I (6.7\%) & I (6.7\%) & $4(26.7 \%)$ \\
\hline I'm afraid of choking on liquids & $5(33.3 \%)$ & I (6.7\%) & - & I (6.7\%) & $8(53.3 \%)$ \\
\hline Never know when I'll choke & $5(33.3 \%)$ & - & - & I (6.7\%) & $9(60 \%)$ \\
\hline \multicolumn{6}{|l|}{ Mental health domain } \\
\hline & I (6.7\%) & I (6.7\%) & $2(13.3 \%)$ & $2(13.3 \%)$ & \\
\hline I have to be very careful when I drink or how to bother me & $2(13.3 \%)$ & & & $3(20 \%)$ & $10(66.7 \%)$ \\
\hline I've been discouraged with my deglutition problem & I $(6.6 \%)$ & & $3(20 \%)$ & $3(20 \%)$ & $8(53.3 \%)$ \\
\hline My deglutition problem frustrates me & $2(13.3 \%)$ & & $2(13.3 \%)$ & I (6.7\%) & $10(66.7 \%)$ \\
\hline I become impatient in dealing with my deglutition problem & $2(13.3 \%)$ & I (6.7\%) & & $3(20 \%)$ & $9(60 \%)$ \\
\hline \multicolumn{6}{|l|}{ Social function domain } \\
\hline I do not go out to eat due to my deglutition problem & I (6.7\%) & $3(20 \%)$ & - & - & I I (73.3\%) \\
\hline My deglutition problem becomes difficult to have a social life & $3(20 \%)$ & I (6.7\%) & - & - & II (73.3\%) \\
\hline $\begin{array}{l}\text { My job or my leisure activities changed by my deglutition } \\
\text { problem }\end{array}$ & $3(20 \%)$ & - & - & $3(20 \%)$ & $9(60 \%)$ \\
\hline Social programs and vacations do not satisfy me because of my & & & & & \\
\hline deglutition problem & $2(13.3 \%)$ & - & - & - & $13(86.7 \%)$ \\
\hline My role with family and friends has changed due to my & & & & & \\
\hline deglutition problem & $2(13.3 \%)$ & I (6.7\%) & - & $3(20 \%)$ & $9(60 \%)$ \\
\hline \multicolumn{6}{|l|}{ Sleep domain } \\
\hline Have trouble sleeping? & $3(20 \%)$ & I (6.7\%) & I (6.7\%) & & $10(66.7 \%)$ \\
\hline Is that a problem to keep sleeping? & $3(20 \%)$ & $2(13.3 \%)$ & $3(20 \%)$ & $2(13.3 \%)$ & $5(33.3 \%)$ \\
\hline \multicolumn{6}{|l|}{ Fatigue domain } \\
\hline Do you feel weak? & & $2(13.3 \%)$ & & $2(13.3 \%)$ & I I (73.3\%) \\
\hline Do you feel tired? & $2(13.3 \%)$ & $4(26.7 \%)$ & I (6.7\%) & $2(13.3 \%)$ & $6(40 \%)$ \\
\hline Do you feel exhausted? & $2(13.3 \%)$ & $4(26.7 \%)$ & I (6.7\%) & I (6.7\%) & $7(46.7 \%)$ \\
\hline
\end{tabular}


used to assess the degree of dysphagia before and after total laryngectomy in 20 patients (9). It was concluded that the "eating in public" and "normal diet" domains worsened in $50 \%$, even 2 years after surgery. The result reinforces the remarkable social impact that the deglutition difficulty has after total laryngectomy (3), even after a long period after treatment. Moreover, it agrees with the results of our research that revealed a moderate impact on "difficulty eating in public" domain.

However, this same work (9), differs from ours with respect to food consistency, but not to other studies using SWAL-QOL. The percentage of subjects with restriction for solids is comparable to that found in a previous study with the same number of individuals (26.3\%) (11). In another study with 12 patients, 4 had restriction (10), but these had the lowest scores, which suggests that the consistency of food interferes with quality of life (10). When considering the small number of volunteers, we decided not to make this comparison for believing that the subgroups would be uneven and the result would not be representative of the entire population.

Despite the severe and moderate impact found in some domains of SWAL-QOL, no volunteers rated their general health less than satisfactory, and previous studies have also found the same pattern $(10,11)$. This finding can be explained by time to clinical stability at the time of answering the questionnaire. It also reveals that, despite the impact caused by deglutition difficulties, this does not interfere negatively in the context of general health from the perspective of the respondent $(10,11)$.

The frequency of deglutition difficulties is greater when surgery is combined with radiotherapy, as observed in the treatment profile of all of our subjects. Researchers recruited 26 patients who underwent only surgery and 95 who underwent surgery combined with radiotherapy. It was found that the deglutition was better in the group whose treatment was surgical alone (14).

Unlike our current findings, those of our previous research (2) did not detect a negative impression of the patients in relation to deglutition after treatment. In that study, another specific instrument was used for assessment of dysphagia, the MD Anderson Dysphagia Inventory. This shows that the choice of the questionnaire to be applied must be judicious as well as the interpretation of data by the multidisciplinary team.

\section{CONCLUSION}

After total laryngectomy, deglutition exerted a severe impact on quality of life in terms of "communication" and "fear" domains and a moderate impact on the "feeding duration" domain. The other domains caused discrete impact or no impact.

\section{THANKS}

The authors thank the National Council for Technology and Scientific Development (CNPq), which provided financial support through the Notice MCT/CNPq/ Health-CT/MS/SCTIE/DECIT no 67/2009 - REBRATS.

\section{REFERENCES}

1. Genden EM, Ferlito A, Rinaldo A, SilverCE, FaganJJ, Suárez $\mathrm{C}$ et al. Recent changes in the treatment of patients with advanced laryngeal cancer. Head Neck.2008;30(1):103-10.

2. Kazi R, Prasad V, Venkitaraman R, Nutting CM, Clarke P, Rhys-Evans P etal. Questionnaire analysis of the swallowingrelated outcomes following total laryngectomy. Clin Otolaryngol. 2006;31(6):525-30,

3. Maclean J, Cotton S, Perry A. Dysphagia following a total laryngectomy: the effect on quality of life, functioning, and psychological well-being. Dysphagia. 2009;24(3):314-21

4. Schindler A, Favero E, Capaccio P, Albera R, Cavalot AL, Ottaviani F. Supracricoid laryngectomy: age influence on long-term functional results. Laryngoscope. 2009;119(6):1218-25.

5. Ward EC, Bishop B, Frisby J, Stevens M. Swallowing outcomes following total laryngectomy and pharingolaryngectomy. Arch Otolaryngol Head Neck Surg. 2002;128(2):181-6.

6. Maclean J, Cotton S, Perry A. Variation in surgical methods used for total laryngectomy in Australia. 2008; J Laryngol Otol. 122(7):728-32.

7. Zotti P, Lugli D, Vaccher E, Vidotto G, Franchin G, Barzan L. The EORTC quality of life questionnaire-head and neck 35 in Italian laryngectomized patients. European organization for research and treatment of cancer. Qual Life Res. 2000;9(10):1147-53

8.Vilaseca I, Chen AY, Backscheider AG. Long-term quality of life after total laryngectomy. Head Neck.2006;28(4):31320.

9. Chone CT, Spina AL, Barcellos IH, Servin HH, Crespo NA. A prospective study of long-term dysphagia following total laryngectomy. B-ENT. 2011;7(2):103-9. 
10. Barros APB, Portas JG, Queija DS, Lehn CN, Dedivitis RA. Autopercepção da desvantagem vocal (VHI) e qualidade de vida relacionada à deglutição (SWAL-QOL) de pacientes laringectomizados totais. Rev Bras Cir Cabeça Pescoço. 2007;36(1):32-7.

11. Queija DS, Portas JG, Dedivitis RA, Lehn CN, Barros APB. Deglutição e qualidade de vida após laringectomia e faringolaringectomia total. Braz J Otorhinolaryngol. 2009; 75(4):556-64.

12. Portas JG. Validação para a língua portuguesa-brasileira dos questionários: qualidade de vida em disfagia (SWALQOL) e satisfação do paciente e qualidade do cuidade no tratamento da disfagia (SWAL-CARE) [dissertação]. São Paulo: Fundação Antônio Prudente; 2009.
13. Moukarbel RV, Doyle PC, YooJH, FranklinJH, Day AMB, Fung K. Voice-related quality of life (V-RQOL) outcomes in laryngectomees. Head Neck. 2011;33(1): 31-6.

14. de Casso C, Slevin NJ, Homer JJ. The impact of radiotherapy on swallowing and speech in patients who undergo total laryngectomy. Otolaryngol Head Neck Surg. 2008;139(6):792-7.

15. Noonan BJ, Hegarty J. The impact of total laryngectomy: the patient's perspective. Oncol Nurs Forum. 2010;37(3):293-301. 